\title{
PSFC/JA-01-20
}

\section{CompactPCI Based Data Acquisition with MDSplus}

\author{
J.A. Stillerman, T.W. Fredian
}

July 2001

\author{
Plasma Science and Fusion Center \\ Massachusetts Institute of Technology \\ Cambridge, MA 02139 USA
}

This work was supported by the U.S. Department of Energy, Cooperative Grant No. DE-FC02-99ER54512. Reproduction, translation, publication, use and disposal, in whole or in part, by or for the United States government is permitted.

Submitted for publication to Fusion Engineering and Design. 


\section{CompactPCI based Data acquisition with MDSplus}

\author{
Joshua A. Stillerman and Thomas W. \\ Fredian \\ MIT Plasma Science and Fusion Center \\ NW17-268 \\ 190 Albany St. \\ Cambridge, MA, USA 02139
}

\section{Abstract}

Alcator C-Mod is incorporating CompactPCI based data acquisition cards into the MDSplus data acquisition system. Each crate includes a diskless computer running the mdsip server from MDSplus under the linux operating system, and one or more digitizer cards with onboard memory. A minimal set of software is downloaded to this computer at boot time allowing it to arm and read out the cards when requested by the host data acquisition computers. This diskless design is very attractive in our high field environment and simplifies the maintenance and configuration of the embedded computers. Separating the digitizers from the data acquisition computers allows the data acquisition to be done in a platform independent way. Ethernet provides robust, inexpensive communications. The initial digitizer cards have thirty-two 16 bit, $250 \mathrm{KHz}$ digitizers, 128 Msamples of memory, two arbitrary waveform generators and 8 programmable digital outputs. CompactPCI provides an attractive alternative to our aging CAMAC based data acquisition equipment.

\section{Introduction}

The data acquisition hardware in use at Alcator C-Mod is predominantly based on the CAMAC standard. It consists of approximately 700 modules of 75 different types in 62 crates on 5 SCSI serial highways. The system is aging, and suffers from both robustness and performance problems. Though we would like to replace it, the large installed base makes it infeasible, both for economic and for technical reasons. Until recently hardware for new diagnostics and diagnostic upgrades was chosen to fit into our CAMAC environment. The existing infrastructure and expertise made other choices too expensive. New applications with requirements for large numbers of channels have made the investigation of other data acquisition platforms attractive. The lower hybrid current drive system will need approximately 400 channels and the replacement of the digitizers for the magnetics diagnostic will require another 200 channels. Once a new system is in place, it can be applied to smaller scale applications at relatively low cost. The alternatives of VME[1], standard PCI, new CAMAC modules, and CompactPCI[2] were investigated, and CompactPCI was chosen for the platform.

\section{Background}

The CAMAC standard[3] was designed in the late 1960s and extensively used in experimental the physics community. The hardware is modular, new devices can be plugged into existing crates and operated fairly independently from the modules in place. A wide range of transient recorders is available with onboard memory to hold recorded events. Many specialized modules, such as phase digitizers, time of flight analyzers and high speed timers have been developed for physics diagnostics applications. Crates can be distributed around an experiment or laboratory, and optically isolated from each other using optical u-ports. The advent block mode transfers in the 1980s and SCSI serial highways in the 1990s allow for theoretical transfer rates of about 3 Mbytes/Second, in practice we can achieve only about $0.5 \mathrm{Mbyte} / \mathrm{second}$ from crates with enhanced mode controllers and 0.08 Mbyte/second from unenhanced crates.

The main technical shortcoming is the interdependence of the crates on a given serial highway. If any crate or optical Uport on a highway fails, none of the devices on that highway can be accessed. There are communication schemes that overcome this, such as Ethernet crate controllers and direct connections from each crate back to the host computers. These will need to be pursued for the ongoing support of the existing equipment.

There are additional factors that make CAMAC unattractive for new applications.

The market for CAMAC equipment is small; this causes prices to be relatively high, and the selection of available modules to be fairly limited. Much of the equipment in use at Alcator C-Mod is no longer manufactured. The communication speed is becoming less adequate as data rates and memory sizes increase. The 
limited processing power available in the crates, both complicate the communication protocols, and restrict the data processing that can be offloaded from the data acquisition computers. There are vendors willing to develop new custom hardware for particular applications, but there is not a lot of externally motivated development of new modules.

CompactPCI was chosen for these new applications after considering VME, standard PCI, and new CAMAC modules. VME was rejected for several reasons. The chassis, and the real-time software that runs on the embedded computers are expensive. Most of the available boards require the embedded computer's memory for data acquisition. The embedded computer required external communication hardware to overcome the backplane's bandwidth limitations and a large amount device specific programming. Finally, the VME standard was developed in 1981 and is starting to show its age. A system based on standard PCI cards initially looked very good. The infrastructure consists of inexpensive personal computers with Ethernet communications. Ethernet provides fast and robust data transfers, and data acquisition boards are available from many manufacturers. The main drawbacks are that the boards and connectors are relatively fragile, and that the computer needs to be removed from the rack and disassembled to access or replace the boards; though CPCI is somewhat more expensive and is not as ubiquitous as standard PCI, it nicely addresses these concerns.

\section{Solution}

The platform we chose is based around a CPCI based computer running linux[4]. A diskless boot scenario was chosen to avoid damage from the high magnetic fields in our environment and to simplify the management of these systems. The operating system and root file system are loaded over Ethernet using etherboot[5] to a ramdisk. The computer in each crate loads the same boot image. The systems differentiate themselves by getting dhcp assigned ip addresses. The operating system image includes the MDSplus[6] client library, a telnet deamon for diagnostic purposes, and will eventually include a web server for access to configuration and status information. Using the same boot image on all of the crates makes upgrades and maintenance very simple.

The support for specific data acquisition boards has been written in the MDSplus built-in interpreter called tdi. This scheme was first developed at EPFL[7] for use on the TCV experiment. The code is written so that it can be used either locally on the CPCI computer, or remotely, driven by a central data acquisition computer. If operated locally, with local storage, one of these crates could be used as a complete standalone data acquisition system. When used remotely, as we plan to do, the crate can be treated as a backplane with an intelligent controller. In this remote model, the users can view the new equipment as a simple collection of digitizers; very similar to the existing CAMAC based modules. The powerful embedded computer allows for the data to be preprocessed while it is being read out. The initial implementation supports both subsampling and digital filtering. The platform is flexible, making it be easy to experiment with a variety of importance sampling schemes.

The user interface for controlling the devices is very similar to the existing MDSplus device support. A board specific setup form is used to specify the hardware and software settings to be used. From the users perspective the only distinction between these new devices and the existing equipment is that an ip node name and board number are used instead of a designation for CAMAC highway, crate and slot.

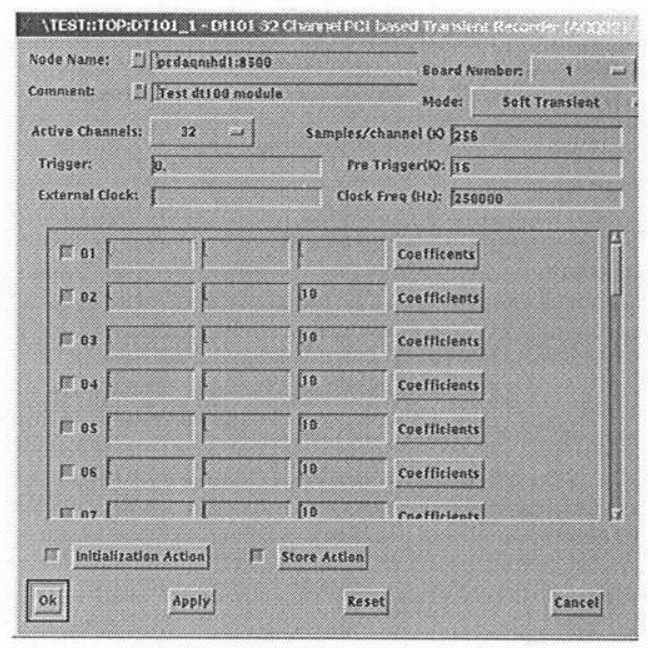

Figure 1 - CPCI device setup 
The initialization routine processes the userspecified settings, optionally connects using mdsip to the remote computer where the hardware is attached, and then evaluates an expression that sets up and arms the hardware. The store routine again optionally connects, and for each channel reads the data, subsampling and digitally filtering them if requested, and writes them to MDSplus. The filters are specified by the user as an array of coefficients to be convolved with the data before they are subsampled. The data can be re-read from the boards at the full time resolution around interesting events. The boards retain the data in their on board memory until the next time they are armed.

\section{Hardware Details}

The first applications of this system are using digitizers purchased from D-Tacq Solutions[8] and timebase decoders being designed by the RFX group at CNR in Padova Italy. The digitizers have thirty-two 16 bit, $250 \mathrm{KHz}$ digitizers, 128 Msamples of memory, two arbitrary waveform generators and 8 programmable digital outputs. Analog input signal processing is done on a mezzanine board, which also houses the front panel and input connectors. The timers are being designed to be backwards compatible with our existing optically distributed timing system[9]. These new timers have 64 bit counters and run at $10 \mathrm{MHz}$ instead of the original modules built by MPB which ran at $1 \mathrm{MHz}$ with 16 bit counters. They will be able to drive the CPCI backplane timing signals directly.

The performance of these digitizers, both in terms of data transfer speed and analog to digital conversion is quite good. The boards can be read by the embedded computer at more than 5 megabytes/second. The data can be read over our local area network at a rate of 2.5 megabytes/second. Figure 2 shows an acquired signal that was digitized at $250 \mathrm{Khz}$. The input signal was a $10 \mathrm{~Hz}$ sine wave with a triangle wave superimposed on it. The added signal's Ofrequency was swept from 3.1 to more than 125 $\mathrm{kHz}$ to see the $50 \mathrm{kHz}$ cutoff frequency of the four pole Butterworth filters in the input circuit. The top panels show the signal sampled at the full rate. The middle panels show the effect of decimating the data to every $10^{\text {th }}$ point. The bottom traces show the effect of subsampling to every $10^{\text {th }}$ point after applying a 41 point digital filter. On the right size of the figure is expanded around at time where the input frequency is about $21 \mathrm{kHz}$, which is well above the Nyquist frequency of the subsampled data. The digital filter attenuates the aliased data by a factor of 100. This clearly demonstrates both the necessity and power of digitally filtering the data when it is being under sampled.

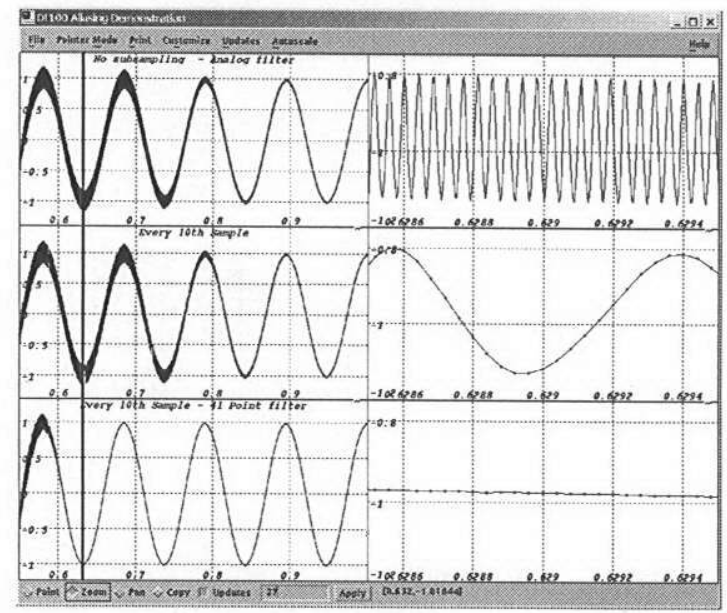

Figure 2 Acquired signals - aliasing and filtering

\section{Conclusion}

CPCI provides an attractive platform for data acquisition under MDSplus. While it would be prohibitively expensive to replace all of our existing CAMAC based equipment, CPCI will work well for new applications and in instances where existing equipment needs to be replaced for maintenance reasons. By choosing diskless linux for the operating system, the crates can be put into place with very low management overhead and should operate robustly in our high magnetic field environment. From the naïve user's perspective this equipment will appear to be very similar to existing device, but embedded computer and network-based communications will provide them significantly better performance.

This work was performed with the support of the Alcator C-Mod staff. Significant contributions were also made by Xavier Llobet and Basil Duval from EPFL, Gabriele Manduchi of CNR and John Mclean and Peter Miline from D-Tacq Solutions. We would like to thank them. 
[1] VITA STANDARDS ORGANIZATION. The VMEbus, Handbook, Fourth Edition, Scottsdale, AZ, (1999)

[2] PCI Industrial Computer Manufacturers Group (PICMG), The CompactPCI Standard, http://www.picmg.org/.

[3]Modular Instrumentation and Interface Standards (CAMAC), ANSI/IEEE Std 583-1982

[4] Linux $(B$ is a registered trademark of Linus Torvalds. http://www.linux.org/

[5] Yap, K., Etherboot: Etherboot home page, http://etherboot.sourceforge.net/

[6] Stillerman,J.A., Fredian,T.W.,Klare,K.A.,Manduchi,G., MDSplus data acquisition system, Review of Scientific Instruments Vol 68 No 1 January 1997 [7] B.P. Duval, et.al., Evolution not Revolution in the TCV Tokamak Control and

Acquisition System, Proceedings of the 21st Symposium on Fusion Technology, Madrid, 11-15 September 2000, to appear.

[8] D-TACQ Solutions Ltd. East Kilbride, SCOTLAND. http://www.d-tacq.com/ [9]V. Schmidt, G. Flor, G. Manduchi, I. Piacentini, "The timing system of the RFX Nuclear Fusion Experiment", Proceeding of the International Conference on Accelerators and Large Experimental Physics Control Systems. KEK, Tsukuba, Japan,November 11 - 15, 1991 pp367-370. 